\title{
Identification of miR-145 as a regulator of the cardiomyocyte inflammatory response and oxidative stress under hyperglycemia
}

\author{
WAN ZHENG, TIANFA LI, JUNPING WEI, YUANYUAN ZHANG, QI ZUO and YUN LIN \\ Department of Cardiovascular Internal Medicine, The First Affiliated Hospital of \\ Hainan Medical University, Haikou, Hainan 570102, P.R. China
}

Received January 16, 2020; Accepted October 23, 2020

DOI: $10.3892 /$ etm.2021.9898

\begin{abstract}
The current study aimed to explore the effects of microRNA (miR)-145 on the inflammatory response and oxidative stress (OS) in high glucose (HG)-induced cardiomyocytes, as well as the specific mechanism underlying this action. H9c2 cells were treated with $33 \mathrm{mmol} / 1$ glucose (HG group) or cotreated with $24.5 \mathrm{mmol} / 1 \mathrm{mannitol}$ and $5.5 \mathrm{mmol} / 1$ glucose (hypertonic group), and the expression levels of miR-145 and ADP ribosylation factor 6 (ARF6) were detected. The cells were transfected with pcDNA3.1-ARF6, miR-145 mimics or corresponding negative controls prior to the assessment of cell survival rate. Levels of lactate dehydrogenase (LDH), reactive oxygen species (ROS) and malondialdehyde (MDA), as well as the activities of superoxide dismutase (SOD), catalase (CAT) and glutathione peroxidase (GPx), and the levels of IL-6, TNF- $\alpha$ and monocyte chemoattractant protein-1 (MCP-1) were subsequently determined. The apoptotic rate of $\mathrm{H} 9 \mathrm{c} 2$ cells was examined by flow cytometry. The interaction between miR-145-ARF6 was predicted and confirmed by luciferase reporter assays. In the HG group, miR-145 expression was significantly decreased and ARF6 expression significantly increased compared with controls. Furthermore, the levels of inflammatory factors (IL-6, TNF- $\alpha$ and MCP-1), LDH, ROS and MDA were significantly elevated in the HG group compared with controls. Significantly decreased SOD, CAT and GPx activities and significantly increased numbers of apoptotic cells were observed in the HG group compared with controls. The cells transfected with miR-145 mimics exhibited significantly decreased LDH, ROS and MDA levels, significantly increased antioxidant enzyme activities and significantly decreased apoptotic rates compared with controls, while the opposite results were observed in cells transfected with pcDNA3.1-ARF6. Moreover, co-transfection
\end{abstract}

Correspondence to: Dr Wan Zheng, Department of Cardiovascular Internal Medicine, The First Affiliated Hospital of Hainan Medical University, 31 Longhua Road, Haikou, Hainan 570102, P.R. China E-mail:meol263@163.com

Key words: high glucose, H9c2, microRNA-145, ADP ribosylation factor 6 , inflammatory response, oxidative stress with miR-145 mimics and pcDNA3.1-ARF6 exacerbated the inflammatory response and OS injury in HG-induced cardiomyocytes compared with cells transfected with miR-145 mimics alone. Furthermore, miR-145 negatively targeted ARF6. miR-145 attenuated the HG-induced inflammatory response and OS injury in cardiomyocytes by negatively regulating ARF6, which may contribute to providing a theoretical basis for the treatment of diabetic cardiomyopathy.

\section{Introduction}

Type 2 difabetes (T2D) is considered a major contributor to cardiovascular disease (CVD), which is the most common cause of T2D-associated death worldwide (1). Diabetic cardiomyopathy, a specific form of CVD, is facilitated by resistance to insulin metabolism in cardiac tissues, compensatory hyperinsulinemia and the progression of hyperglycemia (2). Considering the increasing population of diagnosed, undiagnosed and pre-diagnosed patients with diabetes (3), there is an imperative demand to determine an effective therapy for diabetic cardiomyopathy. Damaged electron transport and hyperglycemia partly result in the increased production of reactive oxygen species (ROS), leading to mitochondrial dysfunction and DNA damage, and consequently causing the development of CVD (4). Imbalance between ROS production and elimination exacerbates oxidative stress (OS) and inflammation (5). OS is hypothesized to be a major damaging factor for insulin resistance, impairment of insulin secretion from $\beta$-cells in pancreatic islets and T2D pathogenesis (including subsequent complications) by inducing several causative factors, including the release of proinflammatory cytokines and the generation of ROS and reactive nitrogen species (6). Additionally, inflammation has been regarded as the critical regulator of atrial fibrillation in obesity and diabetes (4), indicating the role of the inflammatory response in diabetic cardiomyopathy. However, the mechanism underlying the regulation of OS and inflammation in cardiomyocytes remain undetermined.

For years, microRNAs (miRs), which regulate gene expression transcriptionally or post-transcriptionally, have been considered to be novel antioxidants due to their potent regulatory effects on OS (7). For instance, miR-223-3p protects against hypoxia-induced cardiomyocyte apoptosis and OS by targeting Kruppel-like factor 15 (8). Previous data 
has demonstrated that miR-145 serves an important role in the regulation of cardiomyocytes. Geniposide protects $\mathrm{H} 9 \mathrm{c} 2$ cells against lipopolysaccharide (LPS)-induced apoptosis and inflammation by regulating miR-145 expression and the MEK/ERK signaling pathway (9). In human aortic endothelial cells, TNF- $\alpha$ treatment markedly induced the release of inflammatory cytokines, including monocyte chemoattractant protein-1 (MCP-1), IL-6 and IL-8, whilst decreasing the expression of miR-145 (10). Moreover, He et al (11) demonstrated that LPS treatment markedly elevated TNF- $\alpha$ levels, which strongly inhibited miR-145 expression under hyperglycemic conditions. Yuan et al (12) reported that the introduction of miR-145-5p is effective in suppressing the production of inflammatory factors, including IL-1 $\beta$, TNF- $\alpha$ and IL-6 in hypoxia-induced cardiomyocytes. Additionally, an increase in miR-145 ameliorated OS in retinal endothelial cells under hyperglycemic conditions by suppressing ROS generation and malondialdehyde (MDA) activity, and increasing superoxide dismutase (SOD) activity (13). All these results indicated the pivotal role of miR-145 in suppressing inflammation and OS. However, the mechanism of miR-145 on high glucose (HG)-induced OS and the inflammatory response in cardiomyocytes remains to be elucidated.

ADP ribosylation factor 6 (ARF6) is a member of the Ras superfamily of GTP-bound proteins that serves a specific role in the membrane translocation of protein kinase $\mathrm{C} \beta \mathrm{I}$ in glomerular mesangial cells under high-glucose conditions (14). The function of ARF6 is determined by the duration of the GTP-bound active state, which is activated by GTPase-activating protein and GTP-GDP exchange factor (15). In HeLa and H9c2 cells, ARF6 is involved in clathrin-independent endocytosis of the human ether-a-go-go-related gene (16). Notably, miR-145 has been reported to improve macrophage-mediated inflammation by targeting ARF6 (17). The current study used the online database StarBase and dual luciferase reporter assays to evaluate the association between miR-145 and ARF6.

The present study aimed to investigate the mechanism via which miR-145 regulates the inflammatory response and OS injury in cardiomyocytes exposed to HG. The results of the current study demonstrated that the overexpression of miR-145 mitigated the inflammatory response and OS injury induced by $\mathrm{HG}$ treatment in cardiomyocytes. Additionally, the results confirmed that miR-145 exerts its protective role in cardiomyocytes by negatively targeting ARF6. These findings indicated the potential role of miR-145 in the treatment of diabetic cardiomyopathy.

\section{Materials and methods}

Cell culture. H9c2 cardiomyocytes were obtained from the American Type Culture Collection and cultured in complete DMEM (MilliporeSigma) containing 10\% FBS (Gibco; Thermo Fisher Scientific, Inc.), $100 \mathrm{U} / \mathrm{ml}$ penicillin and $100 \mu \mathrm{g} / \mathrm{ml}$ streptomycin. The cells were placed in an incubator at $5 \% \mathrm{CO}_{2}$ and $37^{\circ} \mathrm{C}$.

Cell transfection. $\mathrm{H} 9 \mathrm{c} 2$ cells in the log phase were treated with a high concentration of glucose (33 mmol/l; HG group) or co-treated with mannitol $(24.5 \mathrm{mmol} / \mathrm{l})$ and glucose (5.5 mmol/l) as the control (hypertonic group) for $2 \mathrm{~h}$ at $37^{\circ} \mathrm{C}$.
H9c2 cells in the normal glucose group were treated with a normal concentration of glucose (5.5 mmol/1; NG group) for $2 \mathrm{~h}$ at $37^{\circ} \mathrm{C}$. Following this, cells were transfected with pcDNA3.1-ARF6 (2 $\mu \mathrm{g})$, short hairpin (sh)-ARF6 $(2 \mu \mathrm{g})$, miR-145 mimics (100 $\mathrm{nM})$ or the corresponding negative controls (NCs; pcDNA3.1, sh-NC or mimic NC) using the Lipofectamine ${ }^{\circledR} 2000$ kit (Thermo Fisher Scientific, Inc.) according to the manufacturer's protocol. All transfected materials were obtained from Guangzhou RiboBio Co., Ltd. The sequence of miR-145 mimic was 5'-GUCCAGUUUUCCCAG GAAUCCCU-3' and that of NC mimic was 5'-UUCUCCGAA CGUGUCACGUUU-3'. The cells were accordingly grouped into the NG, hypertonic, $\mathrm{HG}, \mathrm{HG}+$ mimic $\mathrm{NC}, \mathrm{HG}+\operatorname{miR}-145$ mimic, $\mathrm{HG}+$ pcDNA3.1-ARF6, HG + sh-NC, HG + sh-ARF6, $\mathrm{HG}+$ control and $\mathrm{HG}+$ miR-145 mimic + pcDNA3.1-ARF6 groups. Cells in the control group were transfected with pcDNA3.1 and sh-NC (1:1; total mass, $2 \mu \mathrm{g})$. H9c2 cells in the blank group did not receive any treatment. The transfected cells were incubated in serum-free DMEM and subsequently incubated with $5 \% \mathrm{CO}_{2}$ at $37^{\circ} \mathrm{C}$ for $48 \mathrm{~h}$ before later use.

Reverse transcription-quantitative PCR (RT-qPCR). Following transfection, H9c2 cells were lysed in $1 \mathrm{ml} \mathrm{TRIzol}{ }^{\circledR}$ (Thermo Fisher Scientific, Inc.). RNA was extracted in accordance with the instructions of the TRIzol reagent ${ }^{\circledR}$ and reverse transcribed into cDNA using PrimeScript RT reagent kit (Takara Bio, Inc.). The reverse transcription was performed as follows: $50^{\circ} \mathrm{C}$ for $15 \mathrm{~min}, 85^{\circ} \mathrm{C}$ for $5 \mathrm{sec}$ and preservation at $4^{\circ} \mathrm{C}$. The PCR reaction conditions and reaction system were performed using SYBR $^{\circledR}$ Premix Ex Taq ${ }^{\mathrm{TM}}$ II (Takara Bio, Inc.) kit, according to the manufacturer's protocol. An ABI 7500 instrument (AB-4351107; Applied Biosystems; Thermo Fisher Scientific, Inc.) was used for RT-qPCR. The thermocycling conditions were as follows: Pre-denaturation at $95^{\circ} \mathrm{C}$ for $10 \mathrm{~min}$, followed by 40 cycles of denaturation for $10 \mathrm{sec}$ at $95^{\circ} \mathrm{C}$, annealing at $60^{\circ} \mathrm{C}$ for $20 \mathrm{sec}$ and extension at $72^{\circ} \mathrm{C}$ for $34 \mathrm{sec}$. Following this, the expression levels of miR-145 and ARF6 were assessed. All primers for RT-qPCR (Table I) were synthesized by Genewiz, Inc. Relative mRNA and miR expression levels were normalized to GAPDH and U6 levels, respectively. All numerical data were analyzed using the $2^{-\Delta \Delta \mathrm{Cq}}$ method (18). $\Delta \Delta \mathrm{Ct}=[\mathrm{Ct}$ (target gene)-Ct(internal gene)] experimental group-[Ct(target gene)-Ct(internal gene)] control group.

Western blotting. H9c 2 cells were washed with precooled PBS three times at $48 \mathrm{~h}$ post-transfection. Cell lysis buffer [50 mM Tris ( $\mathrm{pH} \mathrm{7.4),} 150 \mathrm{mM} \mathrm{NaCl}$ and 1\% NP-40, 0.1\% SDS; $100 \mu \mathrm{l} / 50 \mathrm{ml}$ ] was added for protein extraction. The lysed cells were placed on ice for $30 \mathrm{~min}$ prior to centrifugation at $13,040 \mathrm{x} \mathrm{g}$ and $4^{\circ} \mathrm{C}$ for $10 \mathrm{~min}$. The supernatant was placed in centrifugation tubes $(0.5 \mathrm{ml})$ and maintained at $-20^{\circ} \mathrm{C}$ or quantified using a BCA kit (Beyotime Institute of Biotechnology). Following quantification, the proteins $(30 \mu \mathrm{g})$ were subjected to denaturation with 6x SDS loading buffer at $100^{\circ} \mathrm{C}$ and separation by $10 \%$ SDS-PAGE electrophoresis. The proteins were then transferred onto PVDF membranes with $4^{\circ} \mathrm{C}$ precooled transfer buffer for $1.5 \mathrm{~h}$ and blocked with $5 \%$ skim milk powder dissolved in TBST for $1 \mathrm{~h}$ at room temperature. The membranes were incubated with TBST containing primary antibodies against ARF6 (cat. no. ab77581; 1:1,000; 
Table I. Primer sequences of the genes.

\begin{tabular}{lll}
\hline Primer & \multicolumn{1}{c}{ Forward sequence $\left(5^{\prime}-3^{\prime}\right)$} & \multicolumn{1}{c}{ Reverse sequence $\left(5^{\prime}-3^{\prime}\right)$} \\
\hline miR-145 & ATCGTCCAGTTTTCCCAGG & CGCCTCCACACACTCACC \\
ARF6 & AGCCGATTCACCATCTTCTATAAC & CAGCATCCTAAACCGCATACC \\
GAPDH & ACCACAGTCCATGCCATCAC & TCCACCACCCTGTTGCTGTA \\
U6 & TCGCTTCGGCAGCACATATAC & GCGTGTCATCCTTGCGCAG \\
\hline
\end{tabular}

miR, microRNA; ARF6, ADP ribosylation factor 6.

Abcam) and $\beta$-actin (cat. no. 4970S; 1:1,000; Cell Signaling Technology, Inc.) at $4^{\circ} \mathrm{C}$ overnight. Following this, membranes were washed three times with TBST (10 min/wash). Goat anti-rabbit IgG antibodies (cat. no. CW0103S, 1:5,000; Beijing ComWin Biotech Co., Ltd.) were added and incubated at room temperature for $2 \mathrm{~h}$. After washing with TBST, the membranes were developed using ECL reagent (Thermo Fisher Scientific, Inc.) and the protein expression levels were detected. To quantify the protein expression, the X-ray films were scanned and analyzed with ImageJ v1.48 software (National Institutes of Health). The experiment was performed in triplicate.

ELISA. Concentrations of IL-6, TNF- $\alpha$ and MCP-1 in the supernatant of H9c2 cells were assessed using Rat IL-6 ELISA Kit (cat.no. ab234570), Rat TNF- $\alpha$ ELISA Kit (cat. no. ab236712) and Rat MCP-1 ELISA Kit (cat. no. ab219045; all from Abcam), according to the manufacturer's protocol.

Lactate dehydrogenase $(L D H)$. An LDH kit (Beyotime Institute of Biotechnology) was used to determine the release of $\mathrm{LDH}$ in $\mathrm{H} 9 \mathrm{c} 2$ cells. The supernatant of $\mathrm{H} 9 \mathrm{c} 2$ cells $(50 \mu \mathrm{l})$ was collected from each well and incubated for $30 \mathrm{~min}$ with reduced nicotinamide adenine dinucleotide and pyruvic acid at $37^{\circ} \mathrm{C}$. Following $\sim 15 \mathrm{~min}, 0.4 \mathrm{~mol} / 1 \mathrm{NaOH}$ was added to stop the reaction. The absorbance values of the samples were measured at $440 \mathrm{~nm}$ using a microplate reader to calculate LDH activity. The absorbance of uncultured cells was detected as the background absorbance value. The maximum LDH content was obtained after the cells were treated with $1 \%$ Triton X-100 (MilliporeSigma) for $60 \mathrm{~min}$ at room temperature.

Detection of ROS. The production of ROS was detected with a fluorescence probe redox-sensitive-fluoroprobe; 2',7'-dichlorofluorescein-diacetate (DCFDA; cat. no. 287810; MilliporeSigma) using a ROS detection kit (cat. no. 88-5930-74; Invitrogen; Thermo Fisher Scientific, Inc.), according to the manufacturer's instructions. DCFDA was diluted with DMEM $(1: 1,000)$ to a final concentration of $10 \mu \mathrm{M}$. After the medium was removed, diluted DCFDA was added until H9c2 cells were fully covered. The cells were then cultured in an incubator with $0.5 \% \mathrm{CO}_{2}$ at $37^{\circ} \mathrm{C}$ in the dark for $1 \mathrm{~h}$ and washed with PBS three times to remove excess DCFDA. Fluorescence intensity was monitored in real time with a laser confocal microscope (magnification, x400; FV1000; Olympus Corporation) at an excitation wavelength of $480 \mathrm{~nm}$ and emission wavelength of $530 \mathrm{~nm}$. The fluorescence intensity was measured as follows: $\lambda \mathrm{ex}=480 / \lambda \mathrm{em}=530 \mathrm{~nm}$.
Detection of MDA. An MDA kit (cat. no. S0131M; Beyotime Institute of Biotechnology) was used for the detection of MDA content in $\mathrm{H} 9 \mathrm{c} 2$ cells according to the manufacturer's protocol. The absorbance of the samples was detected at $532 \mathrm{~nm}$ using a microplate and the content of MDA in the samples was calculated according to the standard curve.

Antioxidant enzyme activities. The activities of SOD, catalase (CAT) and glutathione peroxidase (GPx) in H9c2 cells were detected using commercial detection kits (cat. nos. 706002, 707002 and 703102, respectively; Cayman Chemical Company), according to the manufacturer's protocol. A BCA kit (Beyotime Institute of Biotechnology) was used to determine the concentration of protein in cells. The enzyme activity was expressed based on the content of the original enzymes.

Flow cytometry (FCM). Following transfection, H9c2 cells were washed twice with PBS and digested with pancreatin. Following this, the cells were subjected to centrifugation at 1,200 $\mathrm{x}$ g for $5 \mathrm{~min}$ at room temperature and the supernatant was removed. Subsequently, the cells were resuspended in PBS and centrifuged at 1,200 x $\mathrm{g}$ for $5 \mathrm{~min}$ at room temperature. After the supernatant was removed, the cells $\left(10^{5}-10^{6} / \mathrm{ml}\right)$ were resuspended in $490 \mu \mathrm{l}$ of precooled $1 \mathrm{x}$ binding buffer. Annexin V-FITC (5 $\mu \mathrm{l})$ and propidium iodide (5 $\mu \mathrm{l}$ ) (cat. no. ab14085; Abcam) were added to the cell suspension and mixed. Following 10 min of incubation on ice, cell apoptosis was detected by BD FACSVerse ${ }^{\mathrm{TM}}$ Flow Cytometer (BD Biosciences), and the data were analyzed using FlowJo vX10 software (FlowJo LLC).

Dual luciferase reporter assay. StarBase (http://starbase.sysu. edu.cn/) predicted the potential binding sites between miR-145 and ARF6. According to the prediction, the wild-type (WT) and mutant (MT) sequences of the binding sites between miR-145 and ARF6 were designed separately by Genewiz, Inc. The WT or MT sequence was inserted into a luciferase reporter vector (pGL3-Basic; Promega Corporation) to generate the vectors WT-ARF6 and MT-ARF6. Following this, WT-ARF6 and MT-ARF6 were co-transfected with miR-145 mimics into 293T cells (American Type Culture Collection) using Lipofectamine ${ }^{\circledR} 2000$ kit (Thermo Fisher Scientific, Inc.). At $\sim 48 \mathrm{~h}$ post-transfection, Dual-Luciferase ${ }^{\circledR}$ Reporter Assay System (Promega Corporation) was used to detect the activities of firefly and Renilla luciferase. Renilla luciferase activity was used as the internal control and the ratio between 

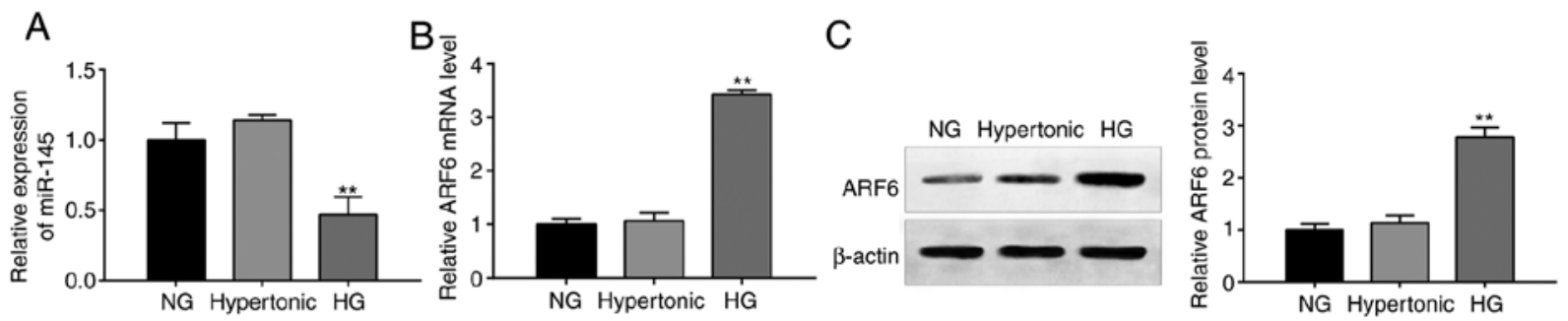

Figure 1. miR-145 and ARF6 expressions are dysregulated in HG-treated H9c2 cells. Cells were treated with 5.5 or 33 mmol/1 glucose, or co-treated with $24.5 \mathrm{mmol} / \mathrm{l}$ mannitol and $5.5 \mathrm{mmol} / \mathrm{l}$ glucose for $2 \mathrm{~h}$. (A) RT-qPCR was performed to detect miR-145 expression. (B) RT-qPCR and (C) western blotting were also performed to measure the mRNA and protein expression of ARF6. ${ }^{* *} \mathrm{P}<0.01$ vs. the NG group. miR, microRNA; ARF6, ADP ribosylation factor 6; HG, high glucose; RT-qPCR, reverse transcription-quantitative PCR; NG, normal glucose.

the activities of firefly luciferase and Renilla luciferase was calculated as the relative activity.

Statistical analysis. Statistical analyses were performed using SPSS (version no. 18.0; SPSS, Inc.) and GraphPad Prism (version no. 6.0; GraphPad Software, Inc.) software. Each experiment was performed in triplicate. Numerical data are presented as the mean \pm standard deviation. Comparisons between two groups were assessed using unpaired Student's t-test, while comparisons among multiple groups conducted using one-way ANOVA with Bonferroni post-hoc analysis. $\mathrm{P}<0.05$ was considered to indicate a statistically significant difference.

\section{Results}

Dysregulation of miR-145 and ARF6 in HG-treated H9c2 cells. RT-qPCR and western blotting revealed that miR-145 expression (Fig. 1A) and the mRNA (Fig. 1B) and protein (Fig. 1C) expression of ARF6 in the hypertonic group differed slightly compared with the NG group; however, this difference was not significant (all, $\mathrm{P}>0.05$ ). In the HG group, miR-145 expression was significantly decreased, while the protein and mRNA expression of ARF6 was significantly increased compared with the NG group (all, $\mathrm{P}<0.01$ ). The results indicated that miR-145 and ARF6 served crucial roles in HG-treated cardiomyocytes.

Protective effect of miR-145 overexpression against the inflammatory response and $\mathrm{OS}$ injury in $\mathrm{HG}$-treated $\mathrm{H} 9 \mathrm{c} 2$ cells. H9c2 cells were transfected with miR-145 mimics or mimic NCs to analyze the effect of miR-145 on HG-treated H9c2 cells. The transfection efficiency of the miR-145 mimics group was detected by RT-qPCR. The results demonstrated that miR-145 expression in the miR-145 mimic group was significantly higher compared with the blank group (Fig. 2A; $\mathrm{P}<0.001)$, indicating the successful transfection of miR-145 mimics.

Compared with the NG group, the levels of IL-6, TNF- $\alpha$ and MCP-1 in the HG group were significantly increased (Fig. 2B-D; $\mathrm{P}<0.01$ ), while the levels of these inflammatory factors were significantly decreased in the $\mathrm{HG}+\mathrm{miR}-145$ mimic group compared with the $\mathrm{HG}+$ mimic $\mathrm{NC}$ group $(\mathrm{P}<0.05)$. The results demonstrated that miR-145 overexpression reduced levels of inflammatory factors in H9c2 cells induced following HG treatment.
The HG group exhibited significantly increased levels of LDH, ROS (Fig. 2E and F; $\mathrm{P}<0.001$ ) and MDA (Fig. 2G; $\mathrm{P}<0.01$ ), as well as significantly decreased activities of $\mathrm{SOD}(\mathrm{P}<0.01)$, CAT $(\mathrm{P}<0.001)$ and GPx $(\mathrm{P}<0.01)$ (Fig. $2 \mathrm{H})$, compared with the NG group. Transfection with miR-145 mimics significantly reduced the levels of $\mathrm{LDH}(\mathrm{P}<0.01)$, MDA $(\mathrm{P}<0.05)$ and ROS $(\mathrm{P}<0.01)$ release, and increased the activities of SOD, CAT and GPx $(\mathrm{P}<0.05)$ in HG-treated H9c2 cells compared with the $\mathrm{HG}+$ mimic NC group. The results indicated that miR-145 overexpression alleviated OS in HG-induced H9c2 cells.

The results of FCM demonstrated that the apoptotic rate of the hypertonic group was not significantly different compared with the NG group (Fig. 2I), while the apoptotic rate of the $\mathrm{HG}$ group was significantly increased compared with the NG group $(\mathrm{P}<0.01)$. The apoptotic rate of the $\mathrm{HG}+\mathrm{miR}-145$ mimic group was significantly lower compared with the $\mathrm{HG}+$ mimic NC group $(\mathrm{P}<0.05)$. Overall, HG treatment induced OS and an inflammatory response in $\mathrm{H} 9 \mathrm{c} 2$ cells, while overexpression of miR-145 attenuated these effects in HG-induced H9c2 cells.

Inhibitory effects of ARF6 silencing on the inflammatory response and $\mathrm{OS}$ injury in $\mathrm{HG}$-treated $\mathrm{H} 9 \mathrm{c} 2$ cells. H9c2 cells were transfected with pcDNA3.1-ARF6, sh-ARF6 or their NCs (pcDNA3.1 or sh-NC, respectively) to investigate the effect of ARF6 on HG-treated H9c2 cells. Following transfection, the mRNA (Fig. 3A) and protein expression (Fig. 3B) of ARF6 was significantly increased in the pcDNA3.1-ARF6 group $(\mathrm{P}<0.001)$ and significantly decreased in the sh-ARF6 group compared with the blank group $(\mathrm{P}<0.01)$, indicating the effective transfection of the ARF6 reagents.

The expression of IL- 6, TNF- $\alpha$ and MCP-1 were significantly increased in the $\mathrm{HG}+$ pcDNA3.1-ARF6 group compared with the $\mathrm{HG}+$ pcDNA3.1 group (Fig. 3C-E; $\mathrm{P}<0.05)$. The expression of these proinflammatory cytokines was also significantly decreased in the $\mathrm{HG}+$ sh-ARF6 group compared with the $\mathrm{HG}+$ sh-NC group (all, $\mathrm{P}<0.01$ ). No significant difference was identified between the $\mathrm{HG}+$ pcDNA3.1 group/HG + sh-NC group and the HG group. The results indicated that ARF6 overexpression increased levels of inflammatory factors and conversely, the inhibition of ARF6 decreased these levels.

Compared with those in the $\mathrm{HG}+$ pcDNA3.1 group, the levels of LDH $(\mathrm{P}<0.05)$, ROS $(\mathrm{P}<0.01)$ release and MDA $(\mathrm{P}<0.05)$ were significantly increased (Fig. 3F-H), while the activities of SOD, CAT and GPx were significantly decreased (Fig. 3I; $\mathrm{P}<0.05$ ) in the $\mathrm{HG}+$ pcDNA3.1-ARF6 group. The 

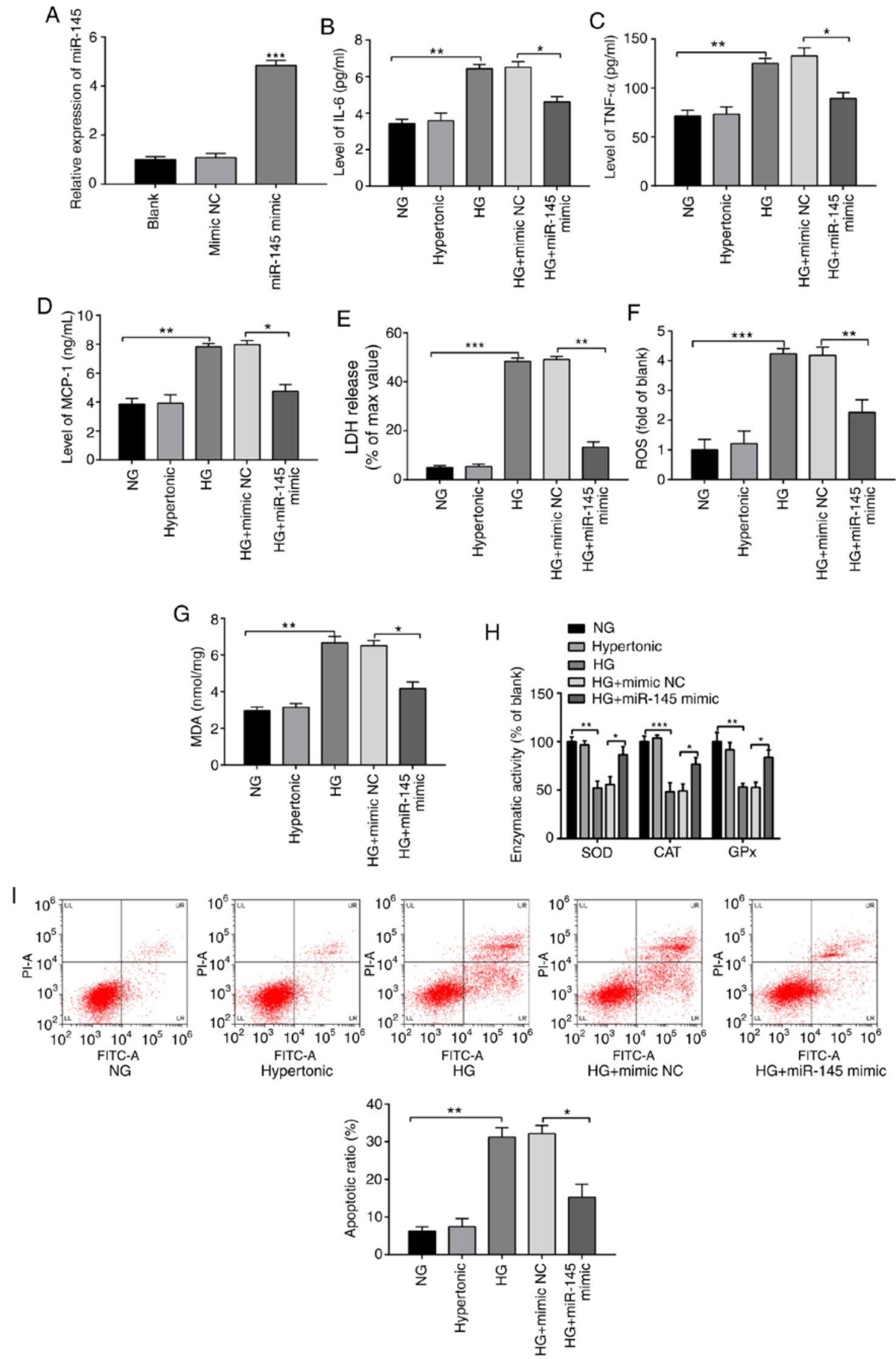

Figure 2. miR-145 ameliorates the inflammatory response and oxidative stress injury in HG-treated H9c2 cells. Following treatment with 5.5 or 33 mmol/1 glucose for $2 \mathrm{~h}, \mathrm{H} 9 \mathrm{c} 2$ cells were transfected with miR-145 mimics or mimic NCs. (A) miR-145 expression was detected by reverse transcription-quantitative PCR. The expression of (B) IL-6, (C) TNF- $\alpha$ and (D) MCP-1 was determined via ELISA. (E) LDH content was detected using an LDH assay. (F) ROS content was examined using an ROS detection kit. (G) ELISA was performed to determine MDA content using a lipid peroxidation (MDA) assay kit. (H) The activities of antioxidant enzymes were evaluated by ELISA. (I) Flow cytometry detected the apoptotic rate of H9c2 cells. ${ }^{*} \mathrm{P}<0.05,{ }^{* *} \mathrm{P}<0.01$ and ${ }^{* * *} \mathrm{P}<0.001$ vs. indicated groups. miR, microRNA; HG, high glucose; NC, negative controls; MCP-1, monocyte chemoattractant protein 1; LDH, lactate dehydrogenase; ROS, reactive oxygen species; MDA, malondialdehyde; NG, normal glucose; SOD, superoxide dismutase; CAT, catalase; GPx, glutathione peroxidase. 

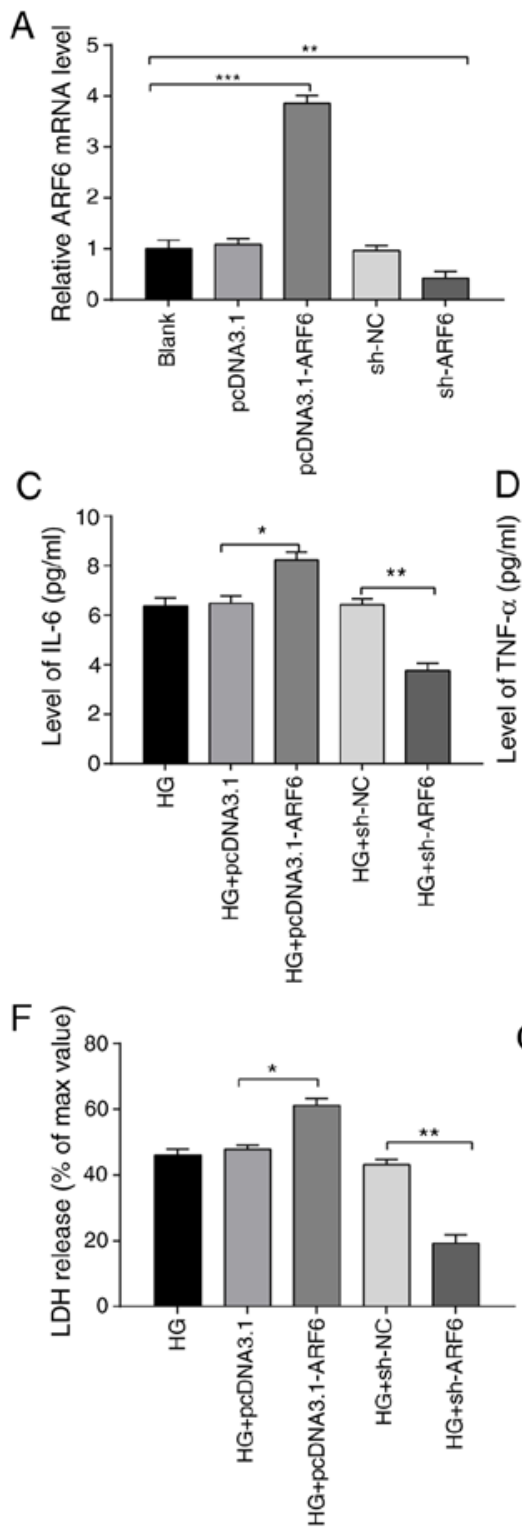
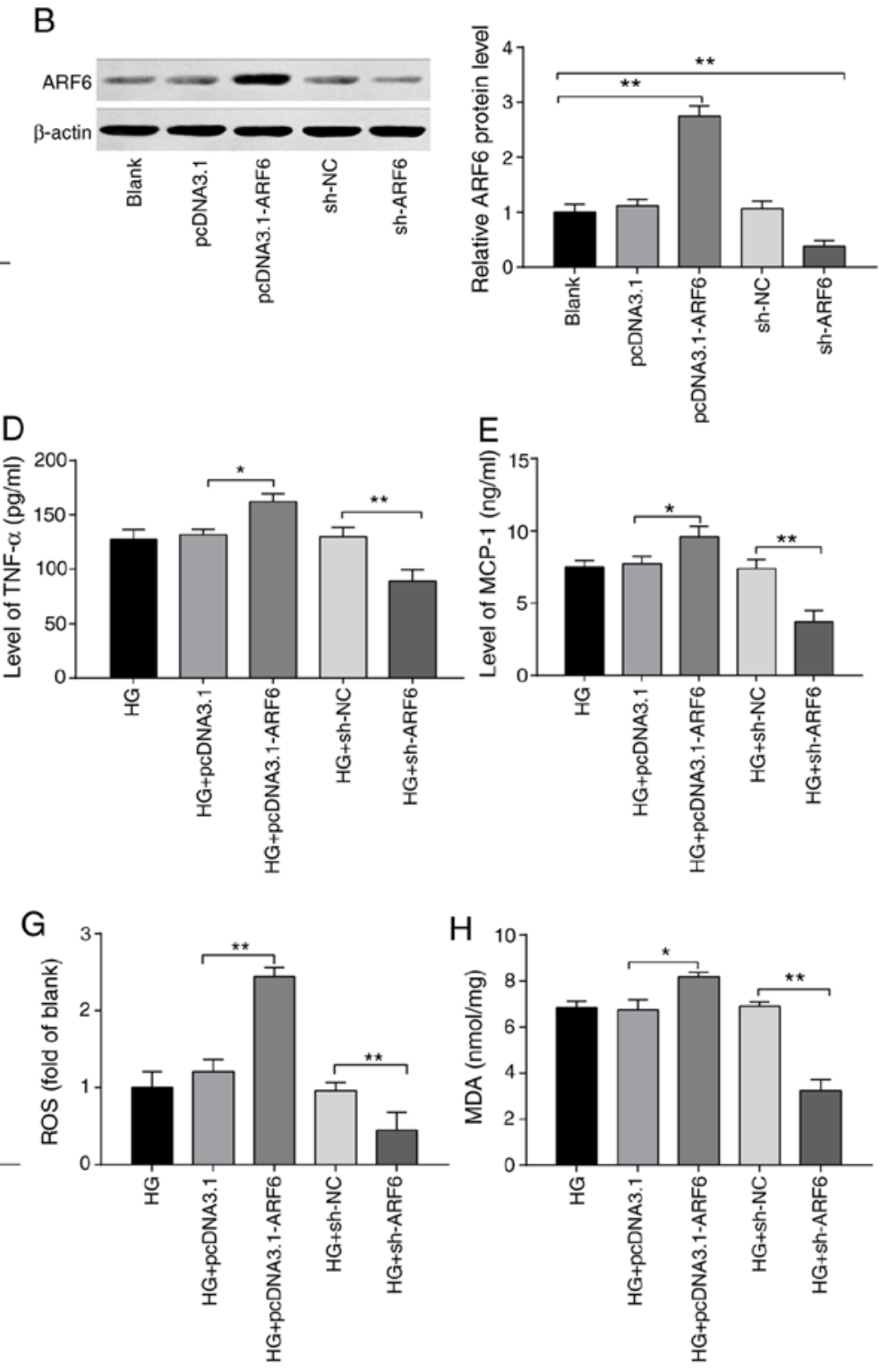

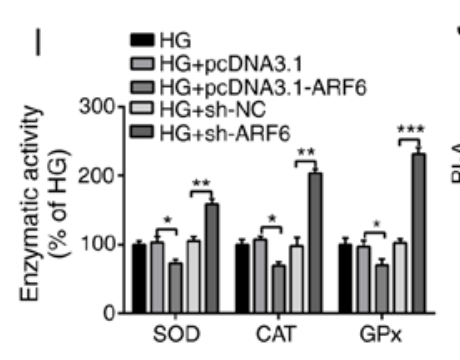

$\mathrm{J}$
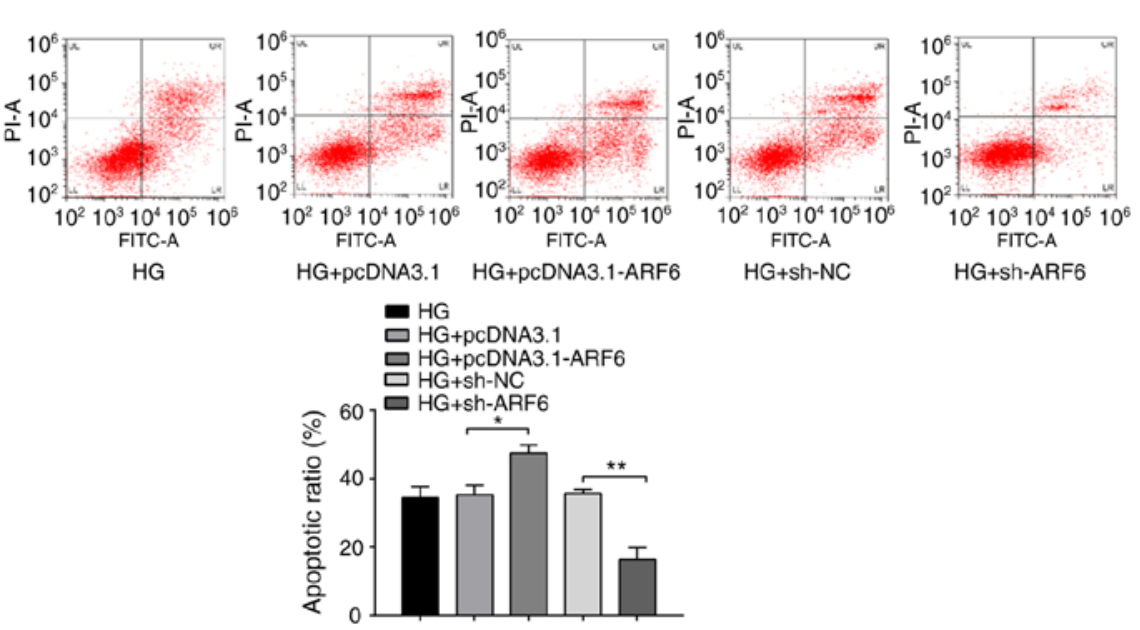

Figure 3. Inhibitory effect of ARF6 knockdown on the inflammatory response and oxidative stress injury in HG-treated H9c2 cells. Following $2 \mathrm{~h}$ of $33 \mathrm{mmol} / \mathrm{l}$ glucose treatment, H9c2 cells were transfected with pcDNA3.1-ARF6, sh-ARF6 or corresponding negative controls, and the transfection efficiency was examined by (A) reverse transcription-quantitative PCR and (B) western blotting. Levels of (C) IL-6, (D) TNF- $\alpha$ and (E) MCP-1 were assessed by ELISA. (F) LDH content was determined via LDH assays. (G) ROS content was measured by ROS kits. (H) MDA content and (I) activities of antioxidant enzymes were evaluated by ELISA. (J) Detection of H9c2 cell apoptotic rate by flow cytometry. ${ }^{*} \mathrm{P}<0.05,{ }^{* *} \mathrm{P}<0.01$ and ${ }^{* * *} \mathrm{P}<0.001$ vs. indicated groups. ARF6, ADP ribosylation factor 6; HG, high glucose; sh, short hairpin; MCP-1, monocyte chemoattractant protein 1; LDH, lactate dehydrogenase; ROS, reactive oxygen species; MDA, malondialdehyde; NC, negative control. 

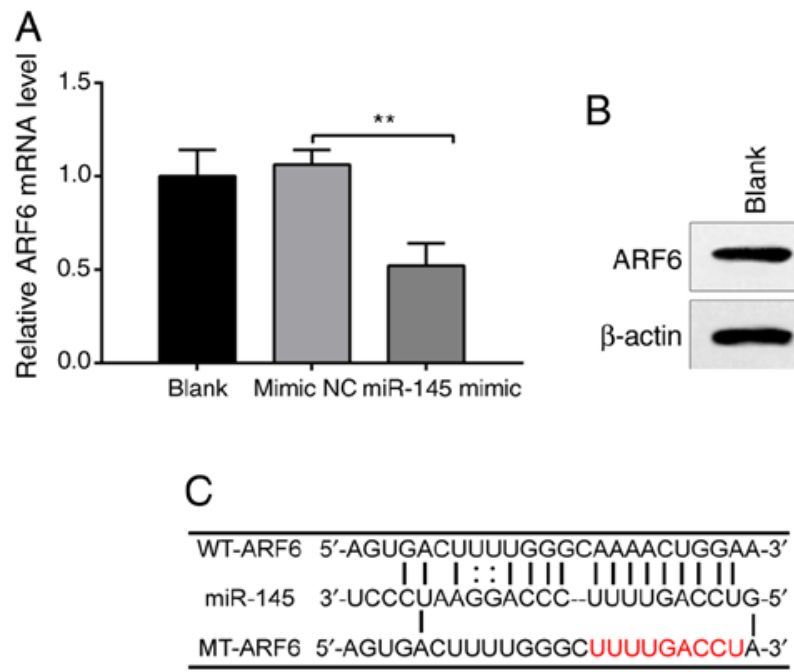
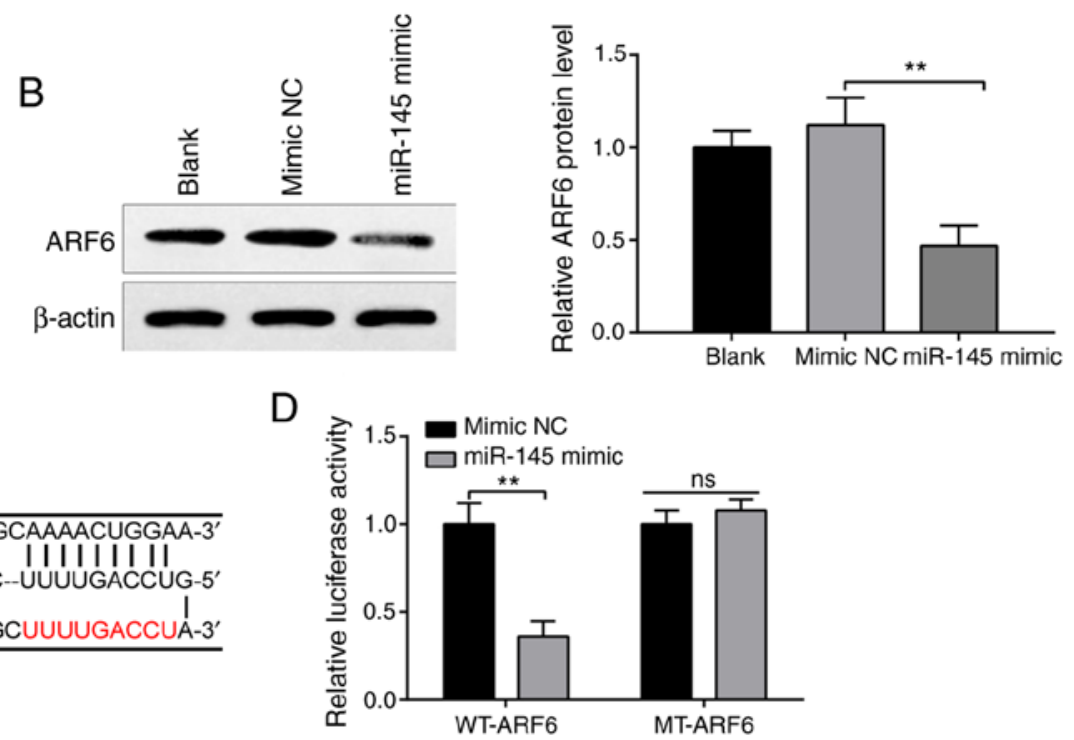

Figure 4. miR-145 negatively regulates ARF6. Following transfection of miR-145 mimics or mimic NCs into H9c2 cells, mRNA and protein expression levels were detected through (A) reverse transcription-quantitative PCR and (B) western blotting. (C) The potential binding sites between ARF6 and miR-145 were predicted by StarBase and WT and MT sites were designed. (D) Detection of the miR-145-ARF6 interaction by luciferase reporter assay following co-transfection of WT-ARF6 or MT-ARF6 with miR-145 mimics or mimic NCs into 293T cells. ${ }^{* *} \mathrm{P}<0.01$ vs. indicated groups. miR, microRNA; ARF6, ADP ribosylation factor 6; NCs, negative controls; WT, wild-type; MT, mutant; ns, not significant.

levels of LDH, ROS and MDA were decreased (Fig. 3F-H; all $\mathrm{P}<0.01)$, while the activities of $\mathrm{SOD}(\mathrm{P}<0.01)$, CAT $(\mathrm{P}<0.01)$ and GPx $(\mathrm{P}<0.001)$ were increased (Fig. 3I) in the $\mathrm{HG}+$ sh-ARF6 group compared with the $\mathrm{HG}+$ sh-NC group. The results confirmed that ARF6 overexpression facilitated OS in $\mathrm{H} 9 \mathrm{c} 2$ cells induced by HG treatment.

Moreover, the apoptotic rate of $\mathrm{H} 9 \mathrm{c} 2$ cells was increased in the $\mathrm{HG}+$ pcDNA3.1-ARF6 group (Fig. 3J; $\mathrm{P}<0.05$ vs. the $\mathrm{HG}+$ pcDNA3.1 group) and decreased in the $\mathrm{HG}+$ sh-ARF6 group $(\mathrm{P}<0.01 \mathrm{vs}$. the $\mathrm{HG}+\mathrm{sh}-\mathrm{NC}$ group). The results indicated that ARF6 overexpression enhanced the inflammatory response and OS injury in HG-treated H9c2 cells, while ARF6 inhibition reversed this effect.

miR-145 negatively targets ARF6. StarBase predicted the potential binding sites between miR-145 and ARF6. H9c2 cells were transfected with miR-145 mimics or mimic NCs, and the expression of ARF6 was measured to investigate the interaction between miR-145 and ARF6. RT-qPCR (Fig. 4A) and western blotting (Fig. 4B) confirmed that transfection with miR-145 mimics significantly decreased the expression of ARF6 compared with the mimic NC group (both $\mathrm{P}<0.01$ ), while ARF6 expression in the mimic NC group was not significantly different compared with the blank group, indicating that miR-145 negatively regulated ARF6.

Accordingly, it was hypothesized that miR-145 inhibited ARF6 expression by binding to the 3' untranslated region (3'UTR) of ARF6. The predicted binding sites between miR-145 and ARF6 and the designed WT and MT sites are presented in Fig. 4C. To test this hypothesis, WT-ARF6 and MT-ARF6 were established. According to the results detected by luciferase reporter assays, co-transfection with WT-ARF6 and miR-145 mimics significantly reduced luciferase activity in 293 T cells compared with the mimic NS group (Fig. 4D; $\mathrm{P}<0.01$, while co-transfection with MT-ARF6 and miR-145 mimic had no significant effect on luciferase activity. These results indicated that miR-145 acted as a sponge of ARF6 and bound to the 3'UTR of ARF6.

miR-145 attenuates the HG-induced inflammatory response and $O S$ injury in cardiomyocytes by regulating ARF6. To confirm the effect of the miR-145-ARF6 interaction on the inflammatory response and OS injury in HG-induced cardiomyocytes, H9c2 cells were transfected with miR-145 mimics, miR-145 mimics + pcDNA3.1-ARF6 or their NCs. RT-qPCR and western blotting was performed to determine the expression levels of miR-145 and ARF6, the results of which exhibited a significant increase in miR-145 expression (Fig. 5A; $\mathrm{P}<0.001$ ) and a significant decrease in ARF6 expression (Fig. 5B and C; $\mathrm{P}<0.01)$ in the $\mathrm{HG}+$ miR-145 mimic group compared with the $\mathrm{HG}+$ control group. No significant difference was observed in miR-145 expression between the HG + miR-145 mimic + pcDNA3.1-ARF6 group and the HG + miR-145 mimic group. However, upregulated ARF6 expression was revealed in the HG + miR-145 mimic + pcDNA3.1-ARF6 group compared with the $\mathrm{HG}+\mathrm{miR}-145$ mimic group at the mRNA and protein expression levels (both, $\mathrm{P}<0.01$ ). The results confirmed successful transfections. Furthermore, the results demonstrated that the expression levels of the inflammatory factors IL-6, TNF- $\alpha$ and MCP-1 in the HG + miR-145 mimic group were significantly decreased (Fig. 5D-F; $\mathrm{P}<0.05$ vs. the $\mathrm{HG}+$ control group) and co-transfection with miR-145 mimics and pcDNA3.1-ARF6 significantly upregulated the expression levels of these inflammatory factors ( $\mathrm{P}<0.05 \mathrm{vs}$. $\mathrm{HG}+\mathrm{miR}-145 \mathrm{mimic}$ group). The levels of these inflammatory factors were not significantly different between the $\mathrm{HG}+$ control and $\mathrm{HG}$ groups. The results suggested that ARF6 overexpression inhibited the effect of miR-145 on reducing the secretion of inflammatory factors in $\mathrm{H} 9 \mathrm{c} 2$ cells.

Moreover, the levels of LDH $(\mathrm{P}<0.05)$, ROS $(\mathrm{P}<0.01)$ and MDA $(\mathrm{P}<0.05)$ were significantly downregulated (Fig. 5G-I) 

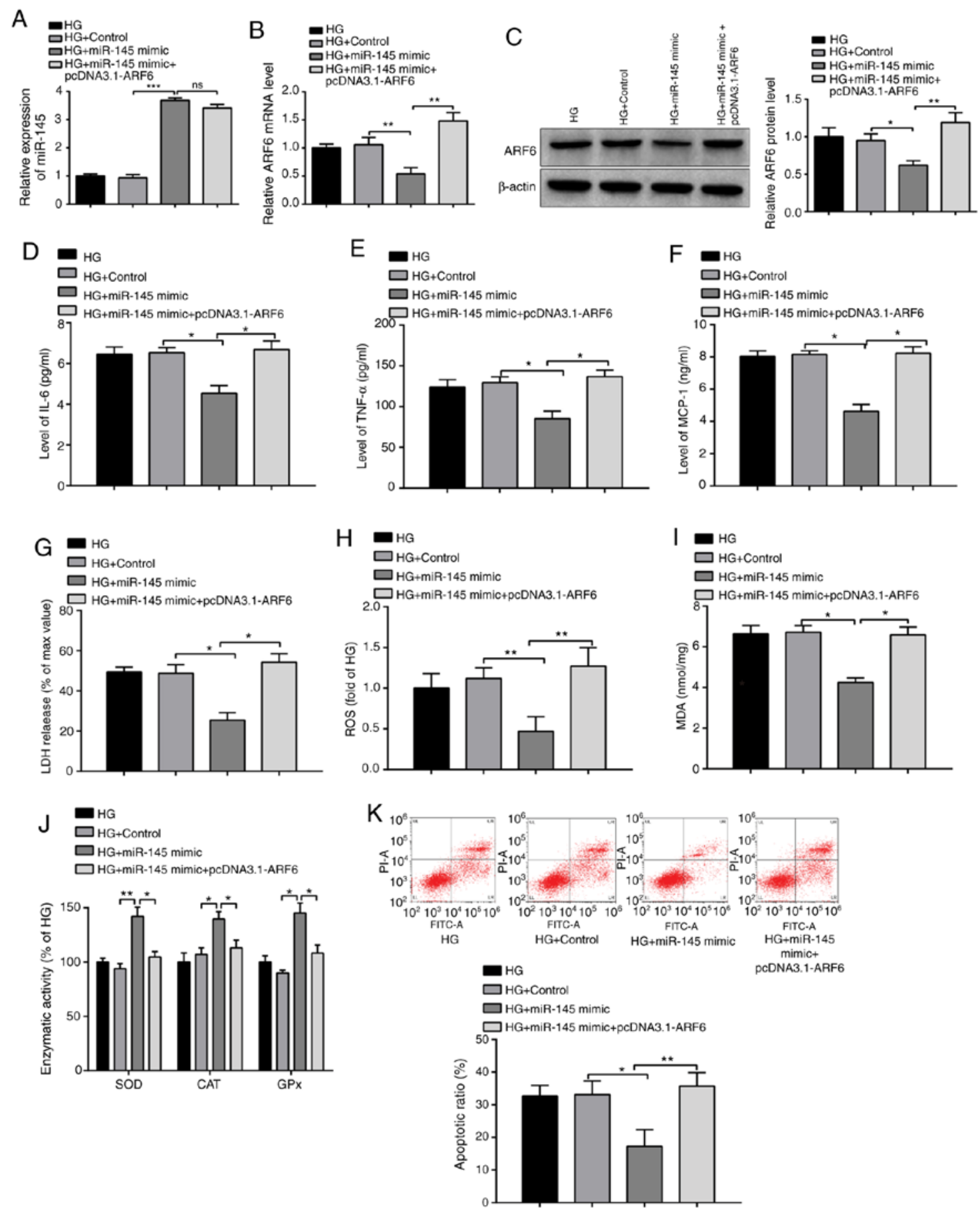

Figure 5. miR-145 protects H9c2 cells from the HG-induced inflammatory response and oxidative stress injury. Following $2 \mathrm{~h}$ of $33 \mathrm{mmol} / \mathrm{l}$ glucose treatment, H9c2 cells were transfected with miR-145 mimics, pcDNA3.1-ARF6, miR-145 + pcDNA3.1-ARF6 or corresponding negative controls, and the expression levels of (A) miR-145 and ARF6 were determined by (B) reverse transcription-quantitative PCR and (C) western blotting. Levels of (D) IL-6, (E) TNF- $\alpha$ and (F) MCP-1 were detected by ELISA. (G) LDH content was detected by a LDH kit. (H) ROS content was determined using an ROS kit. (I) MDA content and $(\mathrm{J})$ activities of antioxidant enzymes were evaluated by ELISA. (K) Detection of H9c2 cell apoptotic rate by flow cytometry. ${ }^{*} \mathrm{P}<0.05,{ }^{* *} \mathrm{P}<0.01$ and ${ }_{* * * *} \mathrm{P}<0.001$ vs. indicated groups. miR, microRNA; HG, high glucose; MCP-1, monocyte chemoattractant protein 1; LDH, lactate dehydrogenase; ROS, reactive oxygen species; MDA, malondialdehyde; ns, not significant.

and the activities of SOD $(\mathrm{P}<0.01)$, CAT $(\mathrm{P}<0.05)$ and GPx $(\mathrm{P}<0.05)$ were significantly upregulated (Fig. 5J) in the $\mathrm{HG}+$ miR-145 mimic group compared with the $\mathrm{HG}+$ control group. Additionally, the levels of LDH $(\mathrm{P}<0.05)$, ROS $(\mathrm{P}<0.01)$ and MDA $(\mathrm{P}<0.05)$ in the $\mathrm{HG}+\mathrm{miR}-145$ mimic + pcDNA3.1-ARF6 group were increased and the activities of the antioxidant enzymes were decreased $(\mathrm{P}<0.05)$ compared with the HG + miR-145 mimic group. The expression levels of these factors were not significantly different between the HG + control and HG groups. These results confirmed that the overexpression of ARF6 reversed the protective effect of miR-145 mimics on HG-induced OS in H9c2 cells.

The apoptotic rate of $\mathrm{H} 9 \mathrm{c} 2$ cells was significantly decreased in the $\mathrm{HG}+$ miR-145 mimic group (Fig. 5K; $\mathrm{P}<0.05$ vs. the $\mathrm{HG}+$ control group) and significantly elevated in the HG + miR-145 mimic + pcDNA3.1-ARF6 group 
$(\mathrm{P}<0.01$ vs. the $\mathrm{HG}+\mathrm{miR}-145$ mimic group). In summary, ARF6 overexpression exacerbated the inflammatory response and $\mathrm{OS}$ injury in HG-induced H9c2 cells, which was suppressed by miR-145 overexpression, supporting the hypothesis that miR-145 alleviated the H9c2 cell inflammatory response and OS injury by regulating ARF6.

\section{Discussion}

miRs, small noncoding RNA molecules whose altered expression has been reported in cardiomyocytes of experimental diabetes models, could be considered as potential treatment targets for diabetic cardiomyopathy in future studies (19). Numerous miRs, such as miR-320, miR-214 and miR-203, have been demonstrated to be associated with diabetes-induced cardiomyopathy and may serve as targets of diabetic cardiomyopathy treatment (20-22). The present study observed downregulated miR-145 and upregulated ARF6 expression in HG-induced cardiomyocytes. Overexpression of miR-145 efficiently protected cardiomyocytes against an HG-induced inflammatory response and OS injury by inhibiting ARF6, indicating a novel therapeutic target against diabetic cardiomyopathy.

Previous studies have extensively documented the antioxidant and anti-inflammatory roles of miR-145. Introduction of miR-145 overexpression in LPS-induced human fibroblast-like synoviocytes inhibited the release of proinflammatory cytokines (23). Injection of miR-145 mimics markedly alleviated mechanical allodynia and thermal hyperalgesia in vivo by suppressing the inflammatory response (24). Upregulation of miR-145 inhibited apoptosis, OS and inflammation in vascular smooth muscle cells, the dominant subunits of the arterial wall, which provided a potential direction for the treatment of abdominal aortic aneurysms (25). The current study demonstrated that miR-145 expression was significantly decreased in response to $\mathrm{HG}$ treatment in cardiomyocytes. Overexpression of miR-145 attenuated OS and the inflammatory response following $\mathrm{HG}$ treatment, as evidenced by significantly decreasing expression levels of IL-6, TNF- $\alpha$, MCP-1, ROS and MDA, as well as significantly increasing activities of SOD, CAT and GPX.

A previous study reported that the inhibition of ARF6 prevented the proinflammatory effects of chloride intracellular channel 4 in human pulmonary artery endothelial cells (26). To date, the role of ARF6 in OS remains undetermined. A previous study indicated that blockade of ARF6 and its effector, c-Myc-binding protein-associated protein-1, increased the generation of ROS in the breast cancer cell line MDA-MB-231 (27). In contrast, a second study demonstrated that ARF6 inhibition induced by secinH3 in insulin-secreting cells (INS-1 832/13 cells) significantly reduced the glucose-induced production of ROS (28). In the current study, ARF6 expression was significantly increased in response to $\mathrm{HG}$ treatment, indicating the involvement of ARF6 in HG-treated cardiomyocytes. Following ARF6 silencing, the inflammatory response and OS injury in cardiomyocytes were alleviated.

ROS activate poly (ADP-ribose) polymerase (PARP)-1 in the nucleus, resulting in the depletion of cellular nicotinamide adenine dinucleotide $\left(\mathrm{NAD}^{+}\right)$and an increase in mitochondrial permeability, mitochondrial dysfunction and cell death (29). PARPs catalyze mono-ADP-ribose or poly-ADP-ribose (PAR) attachment to target proteins via $\mathrm{NAD}^{+}$as a donor (30).
Covalently PARylating target proteins and proteins noncovalently binding with PAR regulate various biological processes, including inflammation (31). The activated GTP-bound form of ARF6 can be ADP-ribosylated on an arginine residue and the ADP ribosylation of ARF6 is an important component of a novel regulatory pathway (32). Therefore, the current study hypothesized that PARP-1 activated by ROS catalyzed the ADP ribosylation of ARF6 GTPase and, therefore, promoted inflammation. Moreover, ARF6 GTPase is required for the polarization of Rabphilin-3A, Ras-related protein Rab-21 and type I phosphatidylinositol 4-phosphate 5-kinase isoform $\gamma$ (PIP5K1C90), and polarization serves an important role in neutrophil adhesion to endothelia during inflammation (33).

The current study hypothesized that miR-145 rescued the inflammatory response and OS injury in HG-treated cardiomyocytes by binding to the 3'UTR of ARF6. The hypothesis was tested using the online database StarBase and dual luciferase reporter assays. miR-145 overexpression reduced the luciferase activity of 293T cells transfected with WT-ARF6; however, this reduction was not observed in cells transfected with MT-ARF6. Therefore, the results confirmed that miR-145 negatively targeted ARF6. Additionally, targeting between miR-145 and ARF6 has been reported in breast cancer (34) and upper tract urothelial carcinoma (35). In subsequent experiments, miR-145 and ARF6 were simultaneously overexpressed to determine the effect of the miR-145-ARF6 interaction on the cardiomyocyte inflammatory response and OS injury in cells treated with HG. The experiments demonstrated that the protective effect of miR-145 overexpression on the HG-induced inflammatory response and OS injury in cardiomyocytes was reversed by ARF6 overexpression, indicating that miR-145 modulated the inflammatory response and OS injury in HG-treated cardiomyocytes by regulating ARF6 expression.

The present study determined that the expression of miR-145 and ARF6 were dysregulated in HG-treated cardiomyocytes. Overexpression of miR-145 or ARF6 silencing in cardiomyocytes attenuated the inflammatory response and OS injury in HG-treated cardiomyocytes. Additionally, the current study demonstrated that miR-145 negatively targeted ARF6, thus protecting cardiomyocytes against the inflammatory response and OS injury induced by HG treatment. The results demonstrated that miR-145 may be used as a novel therapeutic target for diabetic cardiomyopathy. Furthermore, a limitation of the present study was the limited experimental data; therefore, future studies should confirm the current results in animal models.

\section{Acknowledgements}

Not applicable.

\section{Funding}

No funding was received.

\section{Availability of data and materials}

The datasets used and/or analyzed during the current study are available from the corresponding author on reasonable request. 


\section{Authors' contributions}

WZ conceived the study and designed the experiments. TL, JW and YL performed the experiments. WZ, YZ and QZ analyzed the data. QZ, WZ and YL provided critical materials. WZ and TL wrote the manuscript. WZ supervised the study. All authors have read and approved the final manuscript.

\section{Ethics approval and consent to participate}

Not applicable.

\section{Patient consent for publication}

Not applicable.

\section{Competing interests}

The authors declare that they have no competing interests.

\section{References}

1. Newman JD, Vani AK, Aleman JO, Weintraub HS, Berger JS and Schwartzbard AZ: The changing landscape of diabetes therapy for cardiovascular risk reduction: JACC state-of-the-art review. J Am Coll Cardiol 72: 1856-1869, 2018.

2. Jia G, Whaley-Connell A and Sowers JR: Diabetic cardiomyopathy: A hyperglycaemia- and insulin-resistance-induced heart disease. Diabetologia 61: 21-28, 2018.

3. Bugger $\mathrm{H}$ and Abel ED: Molecular mechanisms of diabetic cardiomyopathy. Diabetologia 57: 660-671, 2014.

4. Karam BS, Chavez-Moreno A, Koh W, Akar JG and Akar FG: Oxidative stress and inflammation as central mediators of atrial fibrillation in obesity and diabetes. Cardiovasc Diabetol 16: 120, 2017.

5. Newsholme P, Cruzat VF, Keane KN, Carlessi R and de Bittencourt PI Jr: Molecular mechanisms of ROS production and oxidative stress in diabetes. Biochem J 473: 4527-4550, 2016

6. Qadir MMF, Klein D, Alvarez-Cubela S, Dominguez-Bendala J and Pastori RL: The role of MicroRNAs in diabetes-related oxidative stress. Int J Mol Sci 20: 5423, 2019.

7. Kyrychenko S, Kyrychenko V, Badr MA, Ikeda Y, Sadoshima J and Shirokova N: Pivotal role of miR-448 in the development of ROS-induced cardiomyopathy. Cardiovasc Res 108: 324-334, 2015.

8. Tang Q, Li MY, Su YF, Fu J, Zou ZY, Wang Y and Li SN: Absence of miR-223-3p ameliorates hypoxia-induced injury through repressing cardiomyocyte apoptosis and oxidative stress by targeting KLF15. Eur J Pharmacol 841: 67-74, 2018.

9. Su Q, Yao J and Sheng C: Geniposide attenuates LPS-induced injury via up-regulation of miR-145 in H9c2 cells. Inflammation 41: 1229-1237, 2018.

10. Li L, Liu M, He L, Wang S and Cui S: Baicalin relieves TNF- $\alpha$ evoked injury in human aortic endothelial cells by up-regulation of miR-145. Phytother Res 34: 836-845, 2020.

11. He M, Wu N, Leong MC, Zhang W, Ye Z, Li R, Huang J, Zhang Z Li L, Yao X, et al: miR-145 improves metabolic inflammatory disease through multiple pathways. J Mol Cell Biol 12: 152-162, 2020.

12. Yuan M, Zhang L, You F, Zhou J, Ma Y, Yang F and Tao L: MiR-145-5p regulates hypoxia-induced inflammatory response and apoptosis in cardiomyocytes by targeting CD40. Mol Cell Biochem 431: 123-131, 2017.

13. Hui Y and Yin Y: MicroRNA-145 attenuates high glucose-induced oxidative stress and inflammation in retinal endothelial cells through regulating TLR4/NF- $\kappa \mathrm{B}$ signaling. Life Sci 207: 212-218, 2018

14. Padival AK, Hawkins KS and Huang C: High glucose-induced membrane translocation of PKC betaI is associated with Arf6 in glomerular mesangial cells. Mol Cell Biochem 258: 129-135, 2004.

15. Fang Z, Miao Y, Ding X, Deng H, Liu S, Wang F, Zhou R, Watson $\mathrm{C}, \mathrm{Fu} \mathrm{C}, \mathrm{Hu} \mathrm{Q}$, et al: Proteomic identification and functional characterization of a novel ARF6 GTPase-activating protein, ACAP4. Mol Cell Proteomics 5: 1437-1449, 2006.
16. Karnik R, Ludlow MJ, Abuarab N, Smith AJ, Hardy ME, Elliott DJ and Sivaprasadarao A: Endocytosis of HERG is clathrin-independent and involves arf6. PLoS One 8: e85630, 2013.

17. Li R, Shen Q, Wu N, He M, Liu N, Huang J, Lu B, Yao Q, Yang Y and Hu R: MiR-145 improves macrophage-mediated inflammation through targeting Arf6. Endocrine 60: 73-82, 2018.

18. Livak KJ and Schmittgen TD: Analysis of relative gene expression data using real-time quantitative PCR and the 2(-Delta Delta C(T)) method. Methods 25: 402-408, 2001.

19. Gilca GE, Stefanescu G, Badulescu O, Tanase DM, Bararu I and Ciocoiu M: Diabetic cardiomyopathy: Current approach and potential diagnostic and therapeutic targets. J Diabetes Res 2017: $1310265,2017$.

20. Li H, Fan J, Zhao Y, Zhang X, Dai B, Zhan J, Yin Z, Nie X, Fu XD, Chen $C$ and Wang DW: Nuclear miR-320 mediates diabetes-induced cardiac dysfunction by activating transcription of fatty acid metabolic genes to cause lipotoxicity in the heart. Circ Res 125: 1106-1120, 2019.

21. Wang Y, Zhao RZ, Chen PK, Xu GX, Liu ZJ, Long XP, Qiu ZM and Shi B: Impact and related mechanism on the improvement of hyperglycemia-induced pyroptosis in H9c2 cells by mircoRNA-214. Zhonghua Xin Xue Guan Bing Za Zhi 47: 820-828, 2019 (In Chinese).

22. Yang X, Li X, Lin Q and Xu Q: Up-regulation of microRNA-203 inhibits myocardial fibrosis and oxidative stress in mice with diabetic cardiomyopathy through the inhibition of PI3K/Akt signaling pathway via PIK3CA. Gene 715: 143995, 2019.

23. Zhong F, Xu J, Yang X, Zhang Q, Gao Z, Deng Y, Zhang L and Yu C: miR-145 eliminates lipopolysaccharides-induced inflammatory injury in human fibroblast-like synoviocyte MH7A cells. J Cell Biochem 119: 10059-10066, 2018.

24. Shi J, Jiang K and Li Z: MiR-145 ameliorates neuropathic pain via inhibiting inflammatory responses and mTOR signaling pathway by targeting Akt 3 in a rat model. Neurosci Res 134: $10-17,2018$.

25. Lin H, You B, Lin X, Wang X, Zhou D, Chen Z, Chen Y and Wang R: Silencing of long non-coding RNA Sox 2ot inhibits oxidative stress and inflammation of vascular smooth muscle cells in abdominal aortic aneurysm via microRNA-145-mediated Egr1 inhibition. Aging (Albany NY) 12: 12684-12702, 2020.

26. Abdul-Salam VB, Russomanno G, Chien-Nien C, Mahomed AS, Yates LA, Wilkins MR, Zhao L, Gierula M, Dubois O, Schaeper U, et al: CLIC4/Arf6 pathway. Circ Res 124: 52-65, 2019.

27. Onodera Y, Nam JM, Horikawa M, Shirato H and Sabe H: Arf6-driven cell invasion is intrinsically linked to TRAK1-mediated mitochondrial anterograde trafficking to avoid oxidative catastrophe. Nat Commun 9: 2682, 2018.

28. Jayaram B and Kowluru A: Phagocytic NADPH oxidase links ARNO-Arf6 signaling pathway in glucose-stimulated insulin secretion from the pancreatic $\beta$-cell. Cell Physiol Biochem 30: 1351-1362, 2012.

29. Li C, Zhang J, Xue M, Li X, Han F, Liu X, Xu L, Lu Y, Cheng Y, Li T, et al: SGLT2 inhibition with empagliflozin attenuates myocardial oxidative stress and fibrosis in diabetic mice heart. Cardiovasc Diabetol 18: 15, 2019.

30. Ke Y, Wang C, Zhang J, Zhong X, Wang R, Zeng X and Ba X: The role of PARPs in inflammation-and metabolic-related diseases: Molecular mechanisms and beyond. Cells 8: 1047, 2019.

31. Fehr AR, Singh SA, Kerr CM, Mukai S, Higashi H and Aikawa M: The impact of PARPs and ADP-ribosylation on inflammation and host-pathogen interactions. Genes Dev 34: 341-359, 2020.

32. Dani N, Barbosa AJ, Del Rio A and Di Girolamo M: ADP-ribosylated proteins as old and new drug targets for anticancer therapy: The example of ARF6. Curr Pharm Des 19: 624-633, 2013.

33. Ren C, Yuan Q, Jian X, Randazzo PA, Tang W and Wu D: Small GTPase ARF6 is a coincidence-detection code for RPH3A polarization in neutrophil polarization. J Immunol 204: 1012-1021, 2020.

34. Eades G, Wolfson B,Zhang Y,LiQ, Yao Y and Zhou Q: lincRNA-RoR and miR-145 regulate invasion in triple-negative breast cancer via targeting ARF6. Mol Cancer Res 13: 330-338, 2015.

35. Hsu WC, Li WM, Lee YC, Huang AM, Chang LL, Lin HH, Wu WJ, Li CC, Liang PI and Ke HL: MicroRNA-145 suppresses cell migration and invasion in upper tract urothelial carcinoma by targeting ARF6. FASEB J 34: 5975-5992, 2020.

This work is licensed under a Creative Commons Attribution-NonCommercial-NoDerivatives 4.0 International (CC BY-NC-ND 4.0) License. 